VOL. 47 (1993) [221-231]

\title{
SOCLES OF VERMA MODULES IN QUANTUM GROUPS
}

\author{
A.V. Jeyakumar and P.B. Sarasija
}

In this paper the Verma modules $M_{c}(\lambda)$ over the quantum group $v_{c}(s l(n+1), C)$, where $\varepsilon$ is a primitive $\ell$ th root of 1 are studied. Some commutation relations among the generators of $U_{c}$ are obtained. Using these relations, it is proved that the socle of $M_{c}(\lambda)$ is non-zero.

\section{INTRODUCTION}

A quantum group $U_{q}=U_{q}(g)$ is a $q$-deformation of the classical universal enveloping algebra $U$ of a complex semi-simple Lie algebra $g$, where $q$ is an indeterminate. The representations of $U_{q}$ have recently occupied the attention of many mathematicians (see for example, $[1,2,3,4]$ ). When $q$ is a root of unity, the representation theory of $U_{q}$ has a close bearing on the modular representation theory of semi-simple, simply connected algebraic groups and affine Lie algebras.

In [1], De Concini and Kac defined the notion of Verma modules over $U_{q}$ and $U_{\varepsilon}$ (where $\varepsilon$ is a primitive $\ell$ th root of $1, \ell$ is an odd integer) analogous to the classical Verma modules. In this paper, we study the Verma module $M_{\varepsilon}(\lambda)$ over $U_{\varepsilon}=U_{e}(g)$, where $g=s l(n+1)$, and in particular prove that the socle of $M_{e}(\lambda)$ over $U_{e}$ is nonzero.

\section{Preliminaries}

1.1. Let us fix some notations which are standard (see for example, [1]).

For a fixed $n \in \mathbb{N}$, let $\left(a_{i j}\right)_{1 \leqslant i, j \leqslant n}$ be the cartan matrix of type $A_{n}$.

Let $q$ be an indeterminate and let $A=\mathbb{C}\left[q, q^{-1}\right]$ with the quotient field $\mathbb{C}(q)$. For any integer $M \geqslant 0$, we define

and

$$
[M]=\frac{q^{M}-q^{-M}}{q-q^{-1}} \in A, \quad[M] !=[M][M-1] \ldots[1],
$$

$$
\left[\begin{array}{c}
M \\
j
\end{array}\right]=\frac{[M] !}{[j] ![M-j] !} \quad \text { for } j \in \mathbb{N}, \quad\left[\begin{array}{c}
M \\
0
\end{array}\right]=1 \text {. }
$$

Received 9 March 1992

Copyright Clearance Centre, Inc. Serial-fee code: 0004-9729/93 SA2.00+0.00. 
Let $U_{q}$ be the $\mathbb{C}(q)$ algebra with 1 , defined by the generators $E_{i}, F_{i}, K_{i}^{ \pm 1}$ $(1 \leqslant i \leqslant n)$ with the relations:

$$
\begin{aligned}
& K_{i} K_{i}^{-1}=K_{i}^{-1} K_{i}=1, \quad K_{i} K_{j}=K_{j} K_{i} \\
& K_{i} E_{j} K_{i}^{-1}=q^{a_{i j}} E_{j}, \quad K_{i} F_{j} K_{i}^{-1}=q^{-a_{i j}} F_{j}, \\
& E_{i} F_{j}-F_{j} E_{i}=\delta_{i j} \frac{K_{i}-K_{i}^{-1}}{q-q^{-1}} \\
& E_{i} E_{j}=E_{j} E_{i} \text { if } a_{i j}=0 \\
& E_{i}^{2} E_{j}-\left(q+q^{-1}\right) E_{i} E_{j} E_{i}+E_{j} E_{i}^{2}=0 \text { if } a_{i j}=-1, \\
& F_{i} F_{j}=F_{j} F_{i} \text { if } a_{i j}=0 \\
& F_{i}^{2} F_{j}-\left(q+q^{-1}\right) F_{i} F_{j} F_{i}+F_{j} F_{i}^{2}=0 \text { if } a_{i j}=-1 .
\end{aligned}
$$

Then $U_{\boldsymbol{q}}$ is a Hopf algebra over $\mathbb{C}(q)$ which is called the quantum group associated to the matrix $\left(a_{i j}\right)$, with comultiplication $\triangle$, antipode $S$ and counit $\nu$ defined by

$$
\begin{aligned}
\triangle E_{i} & =E_{i} \otimes 1+K_{i} \otimes E_{i}, \quad \Delta F_{i}=F_{i} \otimes K_{i}^{-1}+1 \otimes F_{i}, \\
\triangle K_{i} & =K_{i} \otimes K_{i} \\
S E_{i} & =-K_{i}^{-1} E_{i}, \quad S F_{i}=-F_{i} K_{i}, \quad S K_{i}=K_{i}^{-1} \\
\nu E_{i} & =0, \quad \nu F_{i}=0, \quad \nu K_{i}=1 .
\end{aligned}
$$

Also introduce the elements

$$
\left[K_{i} ; n\right]=\frac{\left(K_{i} q^{n}-K_{i}^{-1} q^{-n}\right)}{q-q^{-1}} \text { in } U_{q} .
$$

1.2. It is well known that one can introduce a root system associated to the matrix $\left(a_{i j}\right)$. We briefly describe the construction here. For details refer to $[1,5]$.

Let $P$ be a free abelian group with basis $\omega_{i}, i=1,2, \ldots, n$ ( $P$ is usually called the lattice of weights). Let $P^{+}$denote the subgroup of non-negative integral combinations of $\omega_{1}, \omega_{2}, \ldots, \omega_{n}$ and any element of $P^{+}$is called a dominant weight. Define the following elements in $P$ :

let

$$
\begin{aligned}
\rho & =\sum_{i=1}^{n} \omega_{i}, & \alpha_{j} & =\sum_{i=1}^{n} a_{i j} \omega_{i} \quad(j=1, \ldots, n) \\
Q & =\sum_{i} Z \alpha_{i}, & Q_{+} & =\sum_{i} Z_{+} \alpha_{i} .
\end{aligned}
$$

Define a bilinear pairing $P \times Q \rightarrow Z$ by

$$
\left(\omega_{i} \mid \alpha_{j}\right)=\delta_{i j}
$$


Then $\left(\alpha_{i} \mid \alpha_{j}\right)=a_{i j}$, so that we get a symmetric $Z$-valued bilinear form on $Q$ such that $(\alpha \mid \alpha) \in 2 Z$.

Define automorphisms $r_{i}$ of $P$ by $r_{i} \omega_{j}=\omega_{j}-\delta_{i j} \alpha_{i}(i, j=1,2, \ldots, n)$.

Then $r_{i} \alpha_{j}=\alpha_{j}-a_{i j} \alpha_{i}$. Let $W$ be the (finite) subgroup of $G L(P)$ generated by $r_{1}, r_{2}, \ldots, r_{n}$. Then $Q$ is $W$-invariant and the pairing $P \times Q \rightarrow Z$ is $W$-invariant. Let $\Pi=\left\{\alpha_{1}, \alpha_{2}, \ldots, \alpha_{n}\right\}, R=W \Pi$ and denote $R \cap Q_{+}$by $R^{+}$. Then $R$ is a root system corresponding to the cartan matrix $\left(a_{i j}\right)$ with Weyl group $W$ and $R^{+}$the system of positive roots. Clearly $p$ is half the sum of positive roots. We introduce a partial ordering of $P$ by $\lambda \geqslant \mu$ if $\lambda-\mu \in Q_{+}$. Let $w_{0}$ be the unique element of $W$ such that $w_{0}\left(R^{+}\right)=-R^{+}$.

1.3. Let $U_{A}$ be the $A$-subalgebra of $U_{q}$ generated by the elements $E_{i}, F_{i}, K_{i}^{ \pm 1},\left[K_{i} ; 0\right]$ $(i=1,2, \ldots, n)$. Let $U_{A}^{+}$(respectively $U_{A}^{-}$) be the $A$-subalgebra of $U_{A}$ generated by the $E_{i}$ (respectively $F_{i}$ ) and $U_{A}^{0}$ the subalgebra generated by the $K_{i}$ and $\left[K_{i} ; 0\right]$.

1.4. We shall show how to choose a canonical basis for $U_{q}$ from the given set of generators (for details see $[1,5,6]$ ).

We note that we can define an anti-automorphism $\omega$ of $U_{q}$ defined by

$$
\omega E_{i}=F_{i} \quad \omega F_{i}=E_{i}, \quad \omega K_{i}=K_{i}^{-1}, \quad \omega q=q^{-1} .
$$

For any $i, 1 \leqslant i \leqslant n$, there is a unique algebra automorphism $T_{i}$ of $U_{q}$ such that

$$
\begin{aligned}
& T_{i} E_{i}=-F_{i} K_{i}, \quad T_{j} E_{i}=-E_{j} E_{i}+q^{-1} E_{i} E_{j} \text { if } a_{j i}=-1 \\
& \text { and } T_{j}\left(E_{i}\right)=E_{i} \text { if } a_{i j}=0 \\
& T_{i} F_{i}=-K_{i}^{-1} E_{i}, \quad T_{j} F_{i}=-F_{j} F_{i}+q F_{i} F_{j} \text { if } a_{j i}=-1 \\
& \text { and } T_{j}\left(F_{i}\right)=F_{i} \text { if } a_{i j}=0 \\
& T_{i} K_{j}=K_{j} K_{i}^{-a_{i j}}, \quad T_{i} \omega=\omega T_{i} .
\end{aligned}
$$

Let $w \in W$ and let $r_{i_{1}} \ldots r_{i_{k}}$ be a reduced expression of $w$. Then the automorphism $T_{w}=T_{i_{1}} \ldots T_{i_{k}}$ of $U_{q}$ is independent of the choice of the reduced expression of $w$.

Fix a reduced expression $r_{i_{1}} r_{i_{2}} \ldots r_{i_{N}}$ of the longest element of $W$, where $N=$ $\left|R^{+}\right|$. Then this gives us an enumeration of the elements of $R^{+}$:

$$
\beta_{1}=\alpha_{i_{1}}, \quad \beta_{2}=r_{i_{1}} \alpha_{i_{2}}, \ldots, \beta_{N}=r_{i_{1}} \ldots r_{i_{N-1}} \alpha_{i_{N}}
$$


We define the root vectors:

$$
\begin{aligned}
& E_{\beta_{1}}=T_{i_{1}} T_{i_{2}} \ldots T_{i_{s-1}} E_{i_{1}} \text {, } \\
& F_{\beta_{d}}=T_{i_{1}} T_{i_{2}} \ldots T_{i_{\text {a }}} F_{i_{\text {d }}} \quad \text { which is the same as } \omega E_{\beta_{\varepsilon}} \text {. }
\end{aligned}
$$

For $j=\left(j_{1}, j_{2}, \ldots, j_{N}\right) \in Z_{+}^{N}$ let

$$
E^{j}=E_{\beta_{1}}^{j_{1}} \ldots E_{\beta_{N}}^{j_{N}}, \quad F^{j}=\omega E^{j}
$$

The elements $F^{j} K_{1}^{m_{1}} \ldots K_{n}^{m_{n}} E^{r}$ where $j, r \in Z_{+}^{N},\left(m_{1} \ldots m_{n}\right) \in Z^{n}$ form a basis of $U_{q}$ over $\mathbb{C}(q)$.

1.5. Given $\varepsilon \in \mathbb{C}^{*}$, we now consider the specialisation $U_{\varepsilon}=U_{A} /(q-\varepsilon) U_{A}$. We take $\varepsilon$ in such a way that $\varepsilon^{2} \neq 1$.

Then $U_{e}$ is an algebra over $\mathbb{C}$ with generators $E_{i}, F_{i}, K_{i}^{ \pm 1}(1 \leqslant i \leqslant n)$ (identifying these vectors with their images), and defining relations,

$$
\begin{aligned}
& K_{i} K_{j}=K_{j} K_{i}, \quad K_{i} K_{i}^{-1}=K_{i}^{-1} K_{i}=1, \\
& K_{i} E_{j} K_{i}^{-1}=\varepsilon^{a_{i}} E_{j}, \quad K_{i} F_{j} K_{i}^{-1}=\varepsilon^{-a_{i j}} F_{j}, \\
& E_{i} F_{j}-F_{j} E_{i}=\delta_{i j} \frac{K_{i}-K_{i}^{-1}}{\varepsilon-\varepsilon^{-1}}, \\
& E_{i}^{2} E_{j}-\left(\varepsilon+\varepsilon^{-1}\right) E_{i} E_{j} E_{i}+E_{j} E_{i}^{2}=0 \text { if } a_{i j}=-1, \\
& F_{i}^{2} F_{j}-\left(\varepsilon+\varepsilon^{-1}\right) F_{i} F_{j} F_{i}+F_{j} F_{i}^{2}=0 \text { if } a_{i j}=-1, \\
& E_{i} E_{j}=E_{j} E_{i}=0, \quad F_{i} F_{j}=F_{j} F_{i}=0 \text { if } a_{i j}=0 .
\end{aligned}
$$

1.6. We denote by $U_{\varepsilon}^{+}, U_{\varepsilon}^{-}, U_{\varepsilon}^{0}$ the images of $U_{A}^{+}, U_{A}^{-}$, and $U_{A}^{0}$ in $U_{\varepsilon}$. The automorphism $T_{i}$ of $U_{q}$ defined in (1.4) clearly induces an automorphism $T_{i}$ of $U_{e}$. The vectors $E^{j}, F^{j}$ et cetera of (1.4.5) can then be taken to represent their images in $U_{\varepsilon}$. Then the elements $E^{j}, j \in Z_{+}^{N}$ form a basis of $U_{\epsilon}^{+}$over $\mathbb{C}$, and the elements $F^{j} K_{1}^{m_{1}} \ldots K_{n}^{m_{n}} E^{r}$ where $j, r \in Z_{+}^{N}$ and $\left(m_{1} \ldots m_{n}\right) \in Z^{n}$ form a basis of $U_{e}$ over $\mathbb{C}$.

\section{SOME COMMUTATION RELATIONS}

2.1. We shall now introduce certain basic relations among the generators of $U_{\epsilon}$ corresponding to the positive roots.

Consider the following sequence of elements in $U_{\varepsilon}$.

$$
\begin{aligned}
& E_{2}, T_{2} T_{1}\left(E_{2}\right), T_{i+1}\left(E_{i}\right) \quad i=1,2, \ldots, n, \\
& T_{i-2}\left(E_{i}\right) \quad i=3, \ldots, n, T_{i+2} T_{i+1}\left(E_{i}\right) \quad i=1, \ldots, n-2, \\
& \ldots, T_{n} T_{n-1} \ldots T_{2}\left(E_{1}\right) .
\end{aligned}
$$


For convenience we shall write the above terms in the same order.

$$
\begin{aligned}
& E_{2}, E_{1}, E_{i i+1}, \quad i=1,2, \ldots, n, E_{i i-2}, \quad i=3, \ldots, n, \\
& E_{i i+1 i+2}, \quad i=1, \ldots, n-2, \ldots, E_{12 \ldots n} .
\end{aligned}
$$

The subscripts correspond to the various positive roots: For example the subscript 12 corresponds to $\alpha_{1}+\alpha_{2}$, and 123 corresponds to $\alpha_{1}+\alpha_{2}+\alpha_{3}$.

For $A_{2}$ and $A_{3}$ these elements are $E_{2}, E_{1}, E_{12}$ (see [6]) and $E_{2}, E_{1}, E_{3}, E_{12}$, $E_{23}, E_{123}$ respectively.

2.2. Using the identities (1.4.2) we obtain the following commutation formulas among the elements defined in (2.1.2).

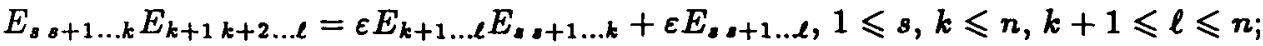

$$
\begin{aligned}
& E_{12 \ldots k} E_{s s+1 \ldots \ell}=E_{s+1 \ldots \ell} E_{12 \ldots k}, 1 \leqslant k \leqslant n, 1<s, \ell<k \text {; } \\
& E_{s,+1 \ldots k} E_{\ell \ell+1 \ldots k}=\varepsilon^{-1} E_{\ell \ell+1 \ldots k} E_{a s+1 \ldots k}, 1 \leqslant s, k \leqslant n, s<\ell \leqslant k \text {; } \\
& E_{s,+1 \ldots k} E_{s,+1 \ldots \ell}=\varepsilon E_{s,+1 \ldots \ell} E_{a,+1 \ldots k}, 1 \leqslant s, k \leqslant n, s \leqslant \ell<k \text {; } \\
& E_{s s+1 \ldots k} E_{\ell \ell+1 \ldots m}=E_{\ell \ell+1 \ldots m} E_{\imath,+1 \ldots k}+\left(\varepsilon^{-1}-\varepsilon\right) E_{r r+1 \ldots p} E_{\imath,+1 \ldots m}, \\
& 1 \leqslant s, k<n, s \neq k, s<\ell \leqslant k<m \leqslant n, \ell=k=r=p, \ell \leqslant r, p \leqslant k, r \neq p \\
& E_{12 \ldots k} E_{k+2}=E_{k+2} E_{12 \ldots k}, \quad 1<k<n-1 \text {; } \\
& E_{i} E_{j}=E_{j} E_{i}, \quad i, j=1,2 \ldots n, \quad i \neq j, a_{i j}=0 \text {. }
\end{aligned}
$$

The above commutation formulas give rise, by induction, to commutation formulas between the basis element of $U_{e}^{+}$.

$$
\begin{aligned}
& E_{s s+1 \ldots k}^{m} E_{k+1 \ldots \ell}^{p}=\varepsilon^{m p} E_{k+1 \ldots \ell}^{p} E_{s, 1 \ldots k}^{m} \\
& \quad+\sum_{j=1}^{\min (m, p)}((p-j) m+j) \\
& \quad\left[\begin{array}{l}
(j] ! \\
j
\end{array}\right]\left[\begin{array}{c}
m \\
j
\end{array}\right] E_{\star .+1 \ldots \ell}^{j} E_{k+1 \ldots \ell}^{p-j} E_{\ldots+1 \ldots k}^{m-j}
\end{aligned}
$$

$1 \leqslant s, k \leqslant n, k+1 \leqslant \ell \leqslant n ;$

$$
\begin{aligned}
& E_{s s+1 \ldots k}^{m} E_{\ell \ell+1 \ldots t}^{u}=E_{\ell \ell+1 \ldots t}^{u} E_{\imath,+1 \ldots k}^{m} \\
& \quad+\sum_{j=1}^{\min (m, u)}(-1)^{j+1}\left(\varepsilon^{-1}-\varepsilon\right)^{j} \varepsilon^{j-1}[j] !\left[\begin{array}{c}
m \\
j
\end{array}\right]\left[\begin{array}{c}
u \\
j
\end{array}\right] E_{r+1 \ldots p}^{j} E_{\imath+1 \ldots k}^{m-j} E_{\ell \ell+1 \ldots t}^{u-j} E_{, .+1 \ldots t}^{j} ;
\end{aligned}
$$

$1 \leqslant s, k<n, s \neq k, s<\ell \leqslant k<t \leqslant n, \ell=k=r=p, \ell \leqslant r, p \leqslant k, r \neq p ;$ $E_{s s+1 \ldots K}^{m} E_{\ell \ell+1 \ldots k}^{p}=\varepsilon^{-m p} E_{\ell \ell+1 \ldots k}^{p} E_{s s+1 \ldots k}^{m}, 1 \leqslant s, k \leqslant n, s<\ell \leqslant k$;

$E_{12 \ldots k}^{m} E_{s s+1 \ldots \ell}^{p}=E_{s,+1 \ldots \ell}^{p} E_{12 \ldots k}^{m}, 1 \leqslant k \leqslant n, 1<s, \ell<k$;

$E_{s s+1 \ldots k}^{m} E_{s,+1 \ldots \ell}^{p}=\varepsilon^{m p} E_{x x+1 \ldots \ell}^{p} E_{s,+1 \ldots k}^{m}, 1 \leqslant s, k \leqslant n, s \leqslant \ell<k$; 
$E_{12 \ldots k}^{m} E_{k+2}^{p}=E_{k+2}^{p} E_{12 \ldots k}^{m}, 1<k<n-1$;

$E_{i}^{m} E_{j}^{p}=E_{j}^{p} E_{i}^{m} \quad$ if $\quad a_{i j}=0, i \neq j, \quad i, j=1 \ldots n$.

By using the relations $\omega E_{i}=F_{i}, \omega \varepsilon=\varepsilon^{-1}$ we obtain similar relations among the $F_{i}$ 's.

\section{Verma modules}

3.1. The notion of Verma modules over $U_{q}$ and $U_{c}$ was introduced by De Concini and $\mathrm{Kac}$ in [1]. In the rest of the paper, we shall be concerned only with Verma modules over $U_{\varepsilon}$, where $\varepsilon$ is a primitive $\ell$ th root of unity. We recapitulate the definition below:

For each $\lambda \in P$ the Verma module $M_{\varepsilon}(\lambda)$ over $U_{e}$ is the vector space $M_{\varepsilon}(\lambda)$ in which there exists a nonzzero distinguished vector $v_{\lambda}$ such that $U_{e}^{+} v_{\lambda}=0, K v_{\lambda}=$ $\varepsilon^{(\lambda \mid \alpha)} v_{\lambda}, K \in U_{e}^{0}$ where $(\mid)$ is the pairing from $P \times W \rightarrow Z$ defined in (1.2) and $\left\{F^{j} v_{\lambda}\left(j \in Z_{+}^{N}\right)\right\}$ is a basis of $M_{\varepsilon}(\lambda)$. Let $L_{\varepsilon}(\lambda)$ denote the unique irreducible quotient of $M_{\varepsilon}(\lambda)$ by its unique maximal submodule.

Then we have

$$
K v_{\lambda}=\varepsilon^{(\lambda \mid \alpha)} v_{\lambda}
$$

Also for each $h=1,2, \ldots, N, F_{h} v_{\lambda}$ is a weight vector of weight $\lambda-\alpha_{h}$ as easily seen below.

$$
\begin{aligned}
K F_{h} v_{\lambda} & =\varepsilon^{-\left(\alpha \mid \alpha_{h}\right)} F_{h} K v_{\lambda} \\
& \left.=\varepsilon^{-\left(\alpha \mid \alpha_{h}\right)} \varepsilon^{(\lambda \mid \alpha)} F_{h} v_{\lambda} \quad \text { (since }\left(\alpha_{h} \mid \alpha\right)=\left(\alpha \mid \alpha_{h}\right)\right) \\
& =\varepsilon^{-\left(\lambda-\alpha_{h} \mid \alpha\right)} F_{h} v_{\lambda} .
\end{aligned}
$$

3.1.2. This shows that for any $r \in Z_{+}, F_{h}^{r} v_{\lambda}$ is a weight vector of weight $\lambda-r \alpha_{h}$ and therefore each $F^{j} v_{\lambda}\left(=F_{i}^{j_{1}} \ldots F_{N}^{j_{N}} v_{\lambda}\right)$ is a weight vector of weight $\lambda-\sum_{h=1}^{N} j_{h} \alpha_{h}$.

3.2 Verma Modules over some subalgebras of $U_{\epsilon}$.

We first define the subalgebras $U_{r}, U_{r}^{+}, U_{r}^{-}$, of $U_{\epsilon}$ generated by

$$
\begin{aligned}
& \left\{F^{j}, \prod_{i=1}^{n} K_{i}^{m_{i}}, E^{r}, 0<j_{i}, r_{i}<\ell^{r}, \quad\left(m_{1} \ldots m_{n}\right) \in Z^{n}\right\}, \\
& \left\{E^{r}, \prod_{i=1}^{n} K_{i}^{m_{i}}, 0<r_{i}<\ell^{r}, \quad\left(m_{1} \ldots m_{n}\right) \in Z^{n}\right\}, \\
& \left\{F^{j}, 0 \leqslant j_{i}<\ell^{r}\right\} \quad \text { respectively. }
\end{aligned}
$$


The set

$$
\left\{F_{1}^{j_{1}} \ldots F_{N}^{j_{N}} K_{1}^{m_{1}} \ldots K_{n}^{m_{n}} E_{1}^{r_{1}} \ldots E_{N}^{r_{N}}, 0 \leqslant j_{i}, r_{i}<\ell^{r},\left(m_{1} \ldots m_{n}\right) \in Z^{n}\right\}
$$

is a basis of $U_{r}$ and the set

$$
\left\{F_{1}^{j_{1}} \ldots F_{N}^{j_{N}}, 0 \leqslant j_{i}<\ell^{r}\right\} \quad \text { is a basis of } U_{r}^{-} \text {. }
$$

We can then define the Verma modules $M_{\varepsilon, r}(\lambda)$ of weight $\lambda$ over $U_{r}$ analogously to $M_{e}(\lambda)$ over $U_{\epsilon}$, that is, there exists a non-zero vector (say) $\widehat{v}_{\lambda}$ such that $U_{r}^{+} \hat{v}_{\lambda}=0$, $K \widehat{v}_{\lambda}=\varepsilon^{(\lambda \mid \alpha)} \widehat{v}_{\lambda}$ for $K \in U_{r}^{0}$ and $\left\{F^{j} \widehat{v}_{\lambda}, 0 \leqslant j_{i}<\ell^{r}\right\}$ form a basis of $M_{e, r}(\lambda)$.

There is a natural injective homomorphism $f_{r}: M_{e, r}(\lambda) \rightarrow M_{e}(\lambda)$ given by

$$
f_{r}\left(F^{j} \widehat{v}_{\lambda}\right)=F^{j} v_{\lambda}
$$

3.3. We next introduce certain elements defined by $I_{r}$ of $U_{\varepsilon}^{-}$, which play an important role in our future study of the socles of Verma modules and homomorphisms between Verma modules.

For each positive integer $r$, let $I_{r}=F_{1}^{\ell^{r}-1} \ldots F_{N}^{\ell^{r}-1}$ which is an element of $U_{r}^{-}$.

It then follows that $I_{r} v_{\lambda}$ is a weight vector of $U_{r} v_{\lambda}$ of weight $\lambda-2(\ell-1) \rho$, where $\rho$ is half the sum of the positive roots.

In fact,

$$
\begin{aligned}
K I_{r} v_{\lambda} & =K F_{1}^{\ell^{r}-1} F_{2}^{\ell^{r}-1} \ldots F_{N}^{\ell^{r}-1} v_{\lambda} \\
& =\varepsilon^{\left(\lambda-\left(\ell^{r}-1\right) \alpha_{1}+\ldots+\alpha_{N} \mid \alpha\right)} F_{1}^{\ell^{r}-1} \ldots F_{N}^{\ell^{r}-1} v_{\lambda} \quad \text { from [3.1.2] } \\
& =\varepsilon^{\left(\lambda-2\left(\ell^{r}-1\right) \rho \mid \alpha\right)} F_{1}^{\ell^{r}-1} \ldots F_{N}^{\ell^{r}-1} v_{\lambda} \\
& =\varepsilon^{(\lambda+2 \rho \mid \alpha)} F_{1}^{\ell^{r}-1} \ldots F_{N}^{\ell^{r}-1} v_{\lambda} \quad\left[\text { since } \varepsilon^{\ell^{r}}=1\right] \\
& =\varepsilon^{(\lambda-2 \ell \rho+2 \rho \mid \alpha)} F_{1}^{\ell^{r}-1} \ldots F_{N}^{\ell^{r}-1} v_{\lambda} \\
& =\varepsilon^{(\lambda-2(\ell-1) \rho \mid \alpha)} F_{1}^{\ell^{r}-1} \ldots F_{N}^{\ell^{r}-1} v_{\lambda} .
\end{aligned}
$$

In particular, when $\lambda=0$, we see that $I_{r} \widehat{v}_{0}$ is a weight vector of $M_{\varepsilon, r}(0)$ with minimal weight $-2(\ell-1) \rho$.

We observe for later use that $I_{r}$ is an integral of $U_{r}^{-}$. In fact, for $\alpha \in R^{+}$and $a \in \mathbb{N}$ such that $0<a<\ell^{r}, R_{\alpha}^{a} I_{r}$ and $I_{r} F_{\alpha}^{a}$ are in $U_{r}^{-}$. Hence $F_{\alpha}^{a} I_{r} \widehat{v}_{0}$ and $I_{r} F_{\alpha}^{a} \widehat{v}_{0}$ are weight vectors of $M_{\ell, r}(0)$ with weight $-2(\ell-1) \rho-a \alpha$. By the minimality of the weight $-2(\ell-1) \rho$, it follows that $F_{\alpha}^{a} I_{r}=I_{r} F_{\alpha}^{a}=0$. This shows that $I_{r}$ is an integral of $U_{r}^{-}$, in other works $u I_{r}=\nu(u) I_{r}$ for all $u \in U_{r}^{-}$, where $\nu: U_{r}^{-} \rightarrow \mathbb{C}$ is the augmentation function. 
3.4 A HOMOMORPHism BeTWEen TWO VERMA Modules. $M_{\varepsilon}(\lambda), M_{\varepsilon}(\mu)$ is a map $\phi: M_{\varepsilon}(\lambda) \rightarrow M_{\varepsilon}(\mu)$ such that $\phi$ is a vector space homomorphism and $\phi(u v)=u \phi(v)$, $u \in U_{\varepsilon}, v \in M_{\varepsilon}(\lambda)$.

LEMma 3.4.1. If $M_{e}(\lambda), M_{e}(\mu)$ are Verma modules over the quantum group $U_{\varepsilon}$, and there is an injective $U_{e}$ module homomorphism $\phi: M_{e}(\lambda) \rightarrow M_{\varepsilon}(\mu)$, then $\lambda=\mu$ and $\phi$ is multiplication by some element of $\mathbb{C}$.

PROOF: Let $v_{\lambda}, v_{\mu}$ be non-zero highest weight vectors of $M_{\varepsilon}(\lambda), M_{\varepsilon}(\mu)$ respectively. Since $v_{\lambda}$ generates $M_{\varepsilon}(\lambda), \psi$ is determined by $\psi\left(v_{\lambda}\right)$. Say $\psi\left(v_{\lambda}\right)=u v_{\mu}$, $u \in U_{e}^{-}$. Now by definition, $U_{e}^{-}$is the union of the subalgebras $U_{r}^{-}$for $r=1,2, \ldots$ and so there is some $r$ for which $u \in U_{r}^{-}$. Since $I_{r}$ is an integral for $U_{r}^{-}$,

$$
\nu(u) I_{r} v_{\mu}=I_{r} u v_{\mu}=I_{r} \psi\left(v_{\lambda}\right)=\phi\left(I_{r} v_{\lambda}\right)
$$

where $\nu: U_{r}^{-} \rightarrow \mathbb{C}$ is the augmentation function and $I_{r} v_{\lambda}$ is an element of the basis for $M_{\varepsilon}(\lambda)$, so is non-zero, and therefore $\nu(u) \neq 0$. But $\psi\left(v_{\lambda}\right)$ must have weight $\lambda$, so $u v_{\mu}$ has weight $\lambda$, which contradicts $\nu(u) \neq 0$ unless $\lambda=\mu$. Since $v_{\mu}$ spans the $\mu$-weight space of $M_{\varepsilon}(\mu), \psi\left(v_{\lambda}\right)=c v_{\mu}=c v_{\lambda}$ for some $c \in \mathbb{C}$, and $\phi$ is just multiplication by c.

\section{Socle of Verma modules}

Denote the socle of the $U_{e}$ module $M_{e}(\lambda)$ by $\operatorname{Soc}\left(M_{\varepsilon}(\lambda)\right)$ and the socle of the $U_{r}$ module $M_{\varepsilon, r}(\lambda)$ by $\operatorname{Soc}\left(M_{\epsilon, r}(\lambda)\right)$.

Since for any $r>0, M_{\varepsilon, r}(\lambda)$ is finite dimensional, clearly $\operatorname{Soc}\left(M_{\varepsilon, r}(\lambda)\right) \neq 0$. It is interesting to note that even for the infinite dimensional module $M_{c}(\lambda)$, its socle is non-zero. We proceed to prove this in this section.

Lemma 4.1. If $0 \neq u \in U_{r}^{-}$for some $r \in \mathbb{N}$, then $U_{r} u$ contains $\mathbb{C} I_{r}$.

ProOF: We shall order the positive roots $\alpha(1), \alpha(2), \ldots, \alpha(N)$ in such a way that if $\alpha(i)+\alpha(j)=\alpha(k)$ then $k<i, j$.

If $0<a<\ell^{r}$ then clearly

$$
F_{\alpha(1)}^{\ell^{r}-1} F_{\alpha(1)}^{a}=F_{\alpha(1)}^{l^{r}-1+a}=0
$$

We shall prove by induction on $i$, with $1 \leqslant i \leqslant N$, that $F_{\alpha(1)}^{l^{r}-1} \ldots F_{\alpha(i)}^{l^{r}-1} F_{\alpha}^{\alpha}=0$ whenever $\alpha \in\{\alpha(1), \ldots, \alpha(i)\}$ and $0<a<\ell^{r}$.

Suppose there exists some $i, 2 \leqslant i \leqslant N$, such that

$$
F_{\alpha(1)}^{L^{r}-1} F_{\alpha(2)}^{l^{r}-1} \ldots F_{\alpha(i-1)}^{l^{r}-1} F_{\alpha}^{\alpha}=0
$$


whenever $\alpha \in\{\alpha(1), \alpha(2), \ldots \alpha(i-1)\}$ and $0<a<\ell^{r}$.

Now, suppose that there is some $\alpha \in\{\alpha(1), \alpha(2), \ldots, \alpha(i)\}$ and choose $a$ such that $0<a<\ell^{r}$.

If $\alpha=\alpha(i)$, then $F_{\alpha(i)}^{\ell^{r}-1} F_{\alpha}^{a}=0$, and so

$$
F_{\alpha(1)}^{\ell^{r}-1} F_{\alpha(2)}^{\ell^{r}-1} \ldots F_{\alpha(i)}^{\ell^{r}-1} F_{\alpha}^{a}=0
$$

If $\alpha \neq \alpha(i)$, then the commutation relations defined in (2.2) imply that

$$
F_{\alpha(1)}^{\ell^{r}-1} \ldots F_{\alpha(i)}^{\ell^{r}-1} F_{\alpha}^{\alpha}
$$

is a sum of elements of the form

$$
F_{\alpha(1)}^{\ell^{r}-1} \ldots F_{\alpha(i-1)}^{\ell^{r}-1} F_{\beta}^{b} u
$$

with $\beta \in\{\alpha(1), \ldots, \alpha(i-1)\}, 0<b<\ell^{r}, u \in U_{e}$ and each element of this form equals 0 by (4.1.1). So (4.1.1) holds for all $i$.

Using this equation together with the commutation relations defined in (2.2), if $1 \leqslant i \leqslant N$ and $0<a<\ell^{r}$, then

$$
\begin{aligned}
& F_{\alpha(1)}^{\ell^{r}-1} F_{\alpha(2)}^{l^{r}-1} \ldots F_{\alpha(i-1)}^{\ell^{r}-1} F_{\alpha(i)}^{a} \\
& \quad-\epsilon^{-1(i-1)\left(\ell^{r}-1\right)} F_{\alpha(i)}^{a} F_{\alpha(1)}^{\ell^{r}-1} \ldots F_{\alpha(i-1)}^{\ell^{r}-1}=0
\end{aligned}
$$

and so if $1 \leqslant i \leqslant N$ and $0<a, b<\ell^{r}$ then

$$
\begin{aligned}
F_{\alpha(i)}^{a} & F_{\alpha(1)}^{l^{r}-1} F_{\alpha(2)}^{l^{r}-1} \ldots F_{\alpha(i-1)}^{l^{r}-1} F_{\alpha(i)}^{b} \\
& =\varepsilon^{-(i-1)\left(l^{r}-1\right) a} F_{\alpha(1)}^{l^{r}-1} \ldots F_{\alpha(i-1)}^{l^{r}-1} F_{\alpha(i)}^{a+b} \\
& =0 \text { if } a+b \geqslant \ell^{r} .
\end{aligned}
$$

Suppose $u$ is a non-zero element of $U_{r}^{-}$. Then by the basis of $U_{\tau}^{-}$the element $u$ is of the form

$$
F_{\alpha(1)}^{a(1)} F_{\alpha(2)}^{a(2)} \ldots F_{\alpha(N)}^{a(N)} \text { with } 0 \leqslant a(1), \ldots, a(N)<\ell^{r}
$$

By repeated use of (4.1.2)

$$
\begin{aligned}
\mathbb{C} F_{\alpha(N)}^{l^{r}-1-a(N)} \ldots F_{\alpha(1)}^{l^{r}-1-\alpha(1)} u & =\mathbb{C} F_{\alpha(N)}^{\ell^{r}-1} \ldots F_{\alpha(1)}^{l^{r}-1} \\
& =\mathbb{C} I_{r} \text { as required }
\end{aligned}
$$


COROLlary 4.2. Let $r$ be a positive integer.

$$
I_{r+1} \in U_{\epsilon} I_{r}
$$

PROOF: Lemma 4.1 implies that $C_{r+1} \subseteq U_{r+1} I_{r}$, so $I_{r+1} \in U_{r+1} I_{r} \subseteq U_{\varepsilon} I_{r}$.

COROLLARY 4.3 .

(i) If $M$ is a non-zero $U_{r}$ submodule of $M_{\varepsilon, r}(\lambda)$ and $\widehat{v}_{\lambda} \in M_{\epsilon, r}(\lambda)$, then $I_{\boldsymbol{r}} \widehat{v}_{\lambda} \in M$.

(ii) If $M$ is a non-zero $U_{\varepsilon}$ submodule of $M_{\varepsilon}(\lambda)$ and $v_{\lambda} \in M_{\varepsilon}(\lambda)$, then $I_{r} v_{\lambda} \in$ $M$ for all $r$.

Proof:

(i) By the basis of $M_{e, r}(\lambda), M$ contains some vector $u \widehat{v}_{\lambda}$ with $u \in U_{r}^{-}$. By Lemma 4.1, $I_{r} \widehat{v}_{\lambda} \in \mathbb{C} I_{r} \widehat{v}_{\lambda} \subseteq U_{r} u \widehat{v}_{\lambda} \subseteq M$.

(ii) By the basis of $M_{\varepsilon}(\lambda), M$ contains some vector $u v_{\lambda}$ with $u \in U_{\varepsilon}^{-}$, hence $u \in U_{r}^{-}$for some $r$.

By Lemma 4.1, $I_{r} v_{\lambda} \in \mathbb{C} I_{r} v_{\lambda} \subseteq U_{\varepsilon} u v_{\lambda} \subseteq M$.

COROLlaRY 4.4. Soc $\left(M_{e, r}(\lambda)\right)$ is simple.

PROOF: $\operatorname{Soc}\left(M_{\epsilon, r}(\lambda)\right)$ is a non-zero $U_{r}$ submodule of $M_{s_{r}}(\lambda)$ and by Corollary 4.3 (i) the submodule $U_{r} I_{r} \widehat{v}_{\lambda}$ is contained in every simple component of $\operatorname{Soc}\left(M_{\varepsilon, r}(\lambda)\right)$ and hence $\operatorname{Soc}\left(M_{\varepsilon, r}(\lambda)\right)$ itself is simple.

Lemma 4.5. Let $\lambda \in P^{+}$, the set of dominant weights. Then for all $r>0$, the highest weight of $\operatorname{Soc}\left(M_{e, r}(\lambda)\right)$ is $w_{o}(\lambda-2(\ell-1) \rho)$ and hence is independent of $r$.

Proof: From (3.3.1), the lowest weight of $M_{\epsilon, r}(\lambda)$ is $\lambda-2(\ell-1) \rho$ for all $r>0$. From Corollary 4.3(i), we have seen that any non-zero submodule of $M_{\varepsilon, r}(\lambda)$ contains $I_{r} \widehat{v}_{\lambda}$. Hence $\operatorname{Soc}\left(M_{e, r}(\lambda)\right)$ contains $I_{r} \widehat{v}_{\lambda}$ whose weight is $\lambda-2(\ell-1) \rho$. Therefore the lowest weight of $\operatorname{Soc}\left(M_{c}, r(\lambda)\right)$ is $\lambda-2(\ell-1) \rho$ for all $r>0$ and hence the highest weight of $\operatorname{Soc}\left(M_{\epsilon_{,} r}(\lambda)\right)$ is $w_{o}(\lambda-2(\ell-1) \rho)=w_{0}(\lambda+2 \rho)$, which is independent of $r$. Hence the result.

We shall proceed to prove our main result concerning the socle of the Verma modules.

THEOREM 4.6. Soc $\left(M_{e}(\lambda)\right)$ is non-zero for all $\lambda \in P^{+}$.

PROOF: Let $v_{\lambda}, \widehat{v}_{\lambda}$ be non-zero highest weight vectors of the Verma modules $M_{\varepsilon}(\lambda)$ over $U_{e}$ and $M_{\epsilon, r}(\lambda)$ over $U_{r}$ respectively. Let $M$ be an arbitrary non-zero $U_{\varepsilon}$ submodule of $M_{\varepsilon}(\lambda)$. Then by Corollary 4.3(ii), $I_{r} v_{\lambda} \in U_{r} u v_{\lambda} \subseteq M$ for all $r$ and hence $U_{\varepsilon} I_{r} v_{\lambda} \subseteq M$. Now, let $I$ denote the submodule $\bigcap_{r>0} U_{\varepsilon} I_{r} v_{\lambda}$ of $M_{\varepsilon}(\lambda)$. 
Replacing $M$ by each simple component of $\operatorname{Soc}\left(M_{e}(\lambda)\right)$, it immediately follows that $\operatorname{Soc}\left(M_{e}(\lambda)\right) \supseteq I$.

We proceed to prove that $I \neq(0)$. Since $M_{e, r}(\lambda)$ is finite dimensional, $\operatorname{Soc}\left(M_{\varepsilon, r}(\lambda)\right) \neq 0$. By Corollary 4.3(i), $\operatorname{Soc}\left(M_{\varepsilon, r}(\lambda)\right)$ is simple and we can take $\operatorname{Soc}\left(M_{e, r}(\lambda)\right)$ to be isomorphic to the simple $U_{r}$ module $L_{e, r}(\mu)$ (where $\mu$ is $\left.w_{o}(\lambda-2(\ell-1) \rho)\right)$. Also by Corollary 4.3(i), Soc $\left(M_{\varepsilon, r}(\lambda)\right)$ contains $I_{r} \hat{v}_{\lambda}$. Therefore there is some $x_{r}$ in $U_{r}$ such that $x_{r} I_{r} \widehat{v}_{\lambda}$ is in the highest weight space of $\operatorname{Soc}\left(M_{\epsilon, r}(\lambda)\right)$. In other words, $x_{r} I_{r} \widehat{v}_{\lambda} \in\left(M_{e, r}(\lambda)\right)^{\mu}$, the $\mu$ th weight space of $M_{\varepsilon, r}(\lambda)$. Now let $f_{r}$ be the injective $U_{r}$ module homomorphism from $M_{\varepsilon, r}(\lambda)$ to $M_{\varepsilon}(\lambda)$ described in (3.2.3), then $f_{r}\left(\widehat{v}_{\lambda}\right)=v_{\lambda}$.

So, $x_{r} I_{r} v_{\lambda}=f_{r}\left(x_{r} I_{r} \widehat{v}_{\lambda}\right) \in M_{\varepsilon}(\lambda)^{\mu}$.

This shows that for each $r$,

$$
U_{\varepsilon} I_{r} v_{\lambda} \cap\left(M_{\varepsilon}(\lambda)\right)^{\mu} \neq(0)
$$

and is a finite dimensional $\mathbb{C}$-vector space (since $\left(M_{\varepsilon}(\lambda)\right)^{\mu}$ is finite dimensional).

From Corollary (4.2), we have the descending chain of submodules

$$
U_{\varepsilon} I_{1} v_{\lambda} \cap\left(M_{\varepsilon}(\lambda)\right) \supseteq U_{\varepsilon} I_{2} v_{\lambda} \cap\left(M_{\varepsilon}(\lambda)\right)^{\mu} \supseteq \ldots
$$

Hence its intersection which is just $I \cap M_{e}(\lambda)^{\mu}$ is non-zero which implies that $I \neq 0$. Since $\operatorname{Soc}\left(M_{\varepsilon}(\lambda)\right) \supseteq I \neq 0$, it follows that $\operatorname{Soc}\left(M_{\varepsilon}(\lambda)\right) \neq 0$.

Hence the theorem.

\section{REFERENCES}

[1] De Concini and V.G. Kac, 'Representations of quantum groups at roof of 1', in Operator algebras, unitary representations, enveloping algebras and invariant theory, Progr. Math. (Paris 1989) 92 (Birkhauser Boston, Boston), pp. 471-506.

[2] V.G. Drinfield, 'Quantum group', Proc. ICM, Berkely (1986), 798-820.

[3] M. Jimbo, 'A $q$-difference analogue of $U(g)$ and the Yang-Baxter equation', Lett. Math. Phys. 10 (1985), 63-69.

[4] G. Lusztig, 'Modular representations and quantum groups', Contemp. Math. 82 (1989), 58-77.

[5] G. Lusztig, 'Finite dimensional Hopf algebras arising from quantum groups', J. Amer. Math. Soc. 3 (1990), 259-296.

[6] G. Lusztig, 'Quantum group at root of 1', Geom. Dedicata (1990). 